\title{
Effect of neurobic exercise on memory enhancement in the elderly with dementia
}

\author{
Saifon Kanthamalee, Kanid Sripankaew \\ School of Nursing, Eastern Asia University, Pathum Thani, Thailand.
}

Correspondence: Saifon Kanthamalee. Address: 29 Karry Place, Parklea, NSW, Australia, 2768. Email: saifon@eau.ac.th

Received: June 23, 2013

DOI : 10.5430/jnep.v4n3p69

Accepted: September 5, 2013

Online Published: October 29, 2013

\section{Abstract}

The objective of this Quasi-experimental research was to examine the effect of Neurobic Exercise on the Memory Enhancement in the Elderly people suffering from Dementia. During January to September 2012, twenty two female older adults suffering from dementia who were residing at Mitraparpsongkoh Foundation, PathumThani Province in Thailand, were purposively selected to participate in this research. Selected participants were involved in Neurobic Exercise Program on every 2 days continuously for 4 weeks. The instruments consisted of a Demographic Data Form, the Mini-Mental State Examination- Thai 2002, the Memory test and a Neurobic Exercise Program. The reliability of the Mini-Mental State Examination- Thai 2002 and the memory test using Kuder- Richadson 20 were.89 and .81 respectively. Data were analyzed using descriptive statistics and Paired t-test. The results revealed that the average memory scores of the experimental group after receiving the Neurobic Exercise Program were statistically and significantly higher than before receiving the Neurobic Exercise Program at a level of $p<.001$. The results of this study could be used as guidelines by health care providers in enhancing the memory retention of the elderly people suffering from dementia and delaying the deterioration of the brain.

\section{Key words}

Neurobic exercise, Memory, The elderly with dementia

\section{Background}

It has been revealed that the number of elderly people suffering from Dementia is rapidly increasing in the present World. The risk of this disease occurs around the age of 60 years and will be doubled in every 5 years by increasing 20 percent in the age of 80 years old and 35 percent in the elders over 85 years old ${ }^{[1]}$. The memory decline in normal ageing related to the reduction of 2,000 million of neurons and $40 \%$ of the brain damage involved in forming memories ${ }^{[2]}$. The Elderly people suffering from dementia have been found of the death of neurons at cortex and hippocampus, an area that produces the chemical acetylcholine which facilitates learning and memory ${ }^{[3]}$. Therefore, the number of dementia patients is higher among the elderly.

The change of the population structure of the ageing population shows the number of people with dementia is increasing as the population gets older. In Thailand, it is estimated that the number of elderly people suffering from Dementia is set to be doubled within the next 20 years, from 229,000 to 450,000 and could exceed one million within the next 50 years ${ }^{[4]}$. The 
mechanism of dementia is gradually progressing. The sufferers will find difficult to live with others due to the memory deficit, losing their ability to make decisions, personality problems and inability to carry out their daily routine activities. Eventually, the sufferers become totally dependents of their family and carers. Because of the behavioral problems and the physical impairments of the elderly with dementia, they become a burden to the caregivers and as a result the caregivers often feel depressive ${ }^{[5]}$. Moreover, the elderly with dementia needed more time of caring from the caregivers, depending on their cognitive status. The sufferers will require close attention. The cost for the caregivers is estimated about 4,0006,000 baths per month ${ }^{[6]}$. If one of the family members has to resign from job to be the caregiver, the loss of income will cause a domestic economic crisis. Therefore, the dementia condition significantly contributes to financial and human costs. In USA, the costs are estimated at US\$604 billion per year ${ }^{[7]}$.

It is clear that the condition of dementia affects the quality of life of the sufferers. Therefore, the prevention and the delaying of dementia need to be seriously considered. The two treatments that can alleviate the development of dementia are use of pharmacologic and non pharmacologic treatments. Non-pharmacologic methods are reality orientation, cognitive training and reminiscence ${ }^{[8]}$. Reality orientation involves giving regular information to elderly people with dementia about times, place to keep them orientated. Cognitive training can improve memory, language and problemsolving ability among the elderly with dementia ${ }^{[8]}$. In addition, Reminiscence enhanced the cognitive ability by using past experiences of the elders such as pictures, music and important persons are brought into their current thoughts ${ }^{[9]}$. Moreover, the study of the memory training program found memory training program was effective in improving memory in the elders ${ }^{[10]}$. Exercise or physical activity such as walking, swimming, etc. can slow down the memory impairment in the elderly with dementia because the regular exercise increases the blood flows to the brain that encourages the development of new brain cells. In Australia it was found that the physical activity provided a modest improvement in cognition over an 18-month follow-up period ${ }^{[11]}$.

Neurobic exercise, a unique brain exercise program based on the latest findings of scientific research ${ }^{[12]}$. The brain exercise program is presenting the combinations of physical senses, including vision, hearing, taste, smell, touch as well as an emotional sense with changing the daily routine regularly. It stimulates neural activity to strengthen and grow brain cells continuously. Neurobics activate neural systems to work and increases blood supply to the brain ${ }^{[12]}$. Neurobic exercise involves directly with the cortex and hippocampus, located at medial temporal lobe that acts as a temporary store for new information ${ }^{[12]}$ and then gradually transfers to permanent storage in the cortex. The sense of smell is involved in emotional memory and store information to improve the memory ${ }^{[13]}$. In addition, Neurobic exercise stimulates pattern of neural activity that creates direct connections between different brain areas, especially cerebral cortex ${ }^{[12,13]}$. Cerebral cortex is the highest developed part of the human brain. It consists of a large number of different areas, each specialized to retrieve, encode and store information from all senses. Furthermore the connections in the areas of cerebral cortex consist of hundreds of neural pathways, which can store memories in limitless combinations, which is due to the complex system and the number of possible combinations of brain's vast path ways ${ }^{[13]}$. Neurobic exercise helps stimulate the nerve cells to produce natural brain nutrients, called neurotrophins that can increase the size and complexity of dendrites to stay younger, and stronger, and improve memory retention ${ }^{[12]}$.

\section{Purposes of the study}

To compare the memory scores in the elderly with dementia before and after receiving the Neurobic Exercise.

\section{Hypothesis of the study}

Memory scores of the experimental group after receiving the Neurobic Exercise to be higher than before receiving the Neurobic Exercise. 


\section{Conceptual framework of the study}

This study of the effect of Neurobic Exercise on the Memory Enhancement in the elderly with dementia was adapted from Katz (1999) ${ }^{[12]}$ and Atkinson \& Shiffrin (1977) ${ }^{[14]}$. Memory process refers to the storage, retention and recall of information which consists of 3 parts including sensory memory, short term memory and long term memory. The Elderly people who were suffering from dementia were found to be caused by the death of neurons at cortex and hippocampus. Therefore, Neurobic exercise is a brain exercise, using six physical senses and encourages non-routine daily activities to stimulate the nerve cells to be strong and grow continuously as well as to reduce the brain shrinkage. After experiencing a stimulus from the senses such as vision, hear, taste, smell, touch and emotion, in an unexpected method at the cerebral cortex which consists of frontal lobe, occipital lobe, parietal lobe, temporal lobe. This stimulus processes information in sensory memories. Then it is transferred to limbic system in hippocampus and maintained indefinitely in short-term memory by rehearsal or repeating the information over and over again. Short term memory has a capability to process and to keep track of studies through rehearsal, encode and retrieve and then it will enter permanent storage in long-term memory. Neurobic Exercise will increase connections between neurons in the brain. Therefore, this certainly improves memory retention.

Figure 1. Memory process model of Atkinson \& Shiffrin, 1977 in Psychology: Principles and applications (p.225) by Worchel, S. \& Shebilske, W. 1989. New Jersey: Prentice Hall.

New Jersey: Prentice Hall and Neurobic Exercise by Katz CL\& Rubin M. 1999. New York: Workman Publishing Company.

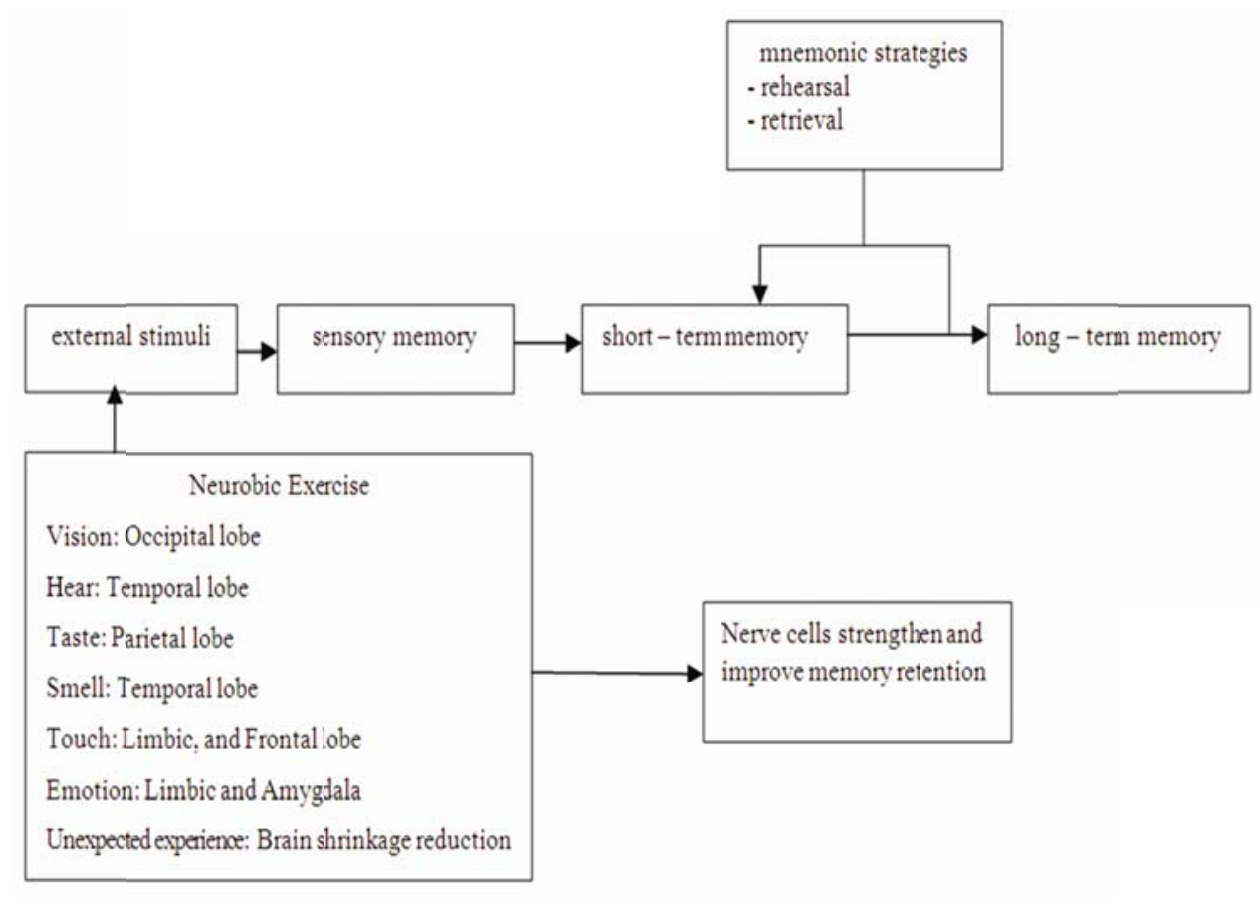

\section{Methodology}

\section{Design}

Quasi-experimental research One Group Pretest-Posttest.

\section{Population and Sample}

Population is the older females who were residing at Mitraparpsongkoh Foundation, PathumThani Province in Thailand. Sample was selected by a purposive sampling from older females who were residing at Mitraparpsongkoh Foundation, PathumThani Province in Thailand during January to September 2012. Twenty two older females were fit for the inclusion criteria of the experimental group who received Neurobic Exercise Program. 


\section{I nclusion criteria}

1) Ability to communicate and understand communication

2) No significant history of psychotropic agents at least 1 week prior to the experiment

3) Not involved in any type of exercises

4) Meeting criteria for dementia; scoring less than or equal 14 in the elders who illiterate, scoring less than or equal 17 in the elders who graduated primary school and scoring less than or equal 22 in the elders who graduated higher than primary school on the Mini-Mental State Examination - Thai 2002.

5) No depressive symptoms which would make reliable assessments difficult, evaluated by depressive evaluation form which should be less than 5 out of 9 .

6) Willing to participate in the experiment.

\section{I nstruments}

The instruments used in this research were data collection instruments and research instruments:

1) Data collection instruments consisted of a Demographic Data Form, the Depression test, the Mini-Mental State Examination- Thai 2002 and the Memory test

2) Research instrument consisted of a Neurobic Exercise Program

\section{Validity and reliability}

The validity of Neurobic Exercise Program was verified by experts. The correction was done as suggested by them. The program was trialed afterwards with the elders similar to the sample group at Thanyaburi Home for the Destitute (Women) to evaluate the true application and objectivity of the language. Kuder-Richadson: KR 20 shows reliabilityof the Mini-Mental State Examination-Thai 2002, the Memory test at.89and .81 respectively.

\section{Data collection procedures}

1) Preceded the data collection by selecting the sample group by a purposive sampling method from the older females suffering from dementia that fitted the inclusion criteria of the experimental group.

2) The researcher explained the purpose and details of the research and requested their cooperation in the research using the declaration of participation in the research and informed the experimental group of the protection of their right in participating in the research.

3) The research explained that the MMSE - Thai 2002 and the Memory tests would be performed on the elders prior to Neurobic Exercise Program.

4) Divided the experimental members into 2 groups with 11 members in each group.

5) Members in each group received Neurobic Exercise Program by using 6 senses such as seeing, hearing, tasting, smelling, touching and emotional sense in combining two or more senses a day. For example: listening and smelling, touching and seeing or smelling, seeing and tasting as well as playing card, digits recall, calculating in mind, asking time and place, phrase repetition and identifying photos. In addition, they were subjected to indulge their daily routine activities such as brushing teeth, writing, combing and eating with an unfamiliar hand or 
changing the sequence of daily activities. For example: having dinner before taking a shower or changing the places where they usually have their meals.

6) Neurobic Exercise Program was given to the experimental group on every 2 days continuously for 4 weeks by researcher. The researcher conducted the training session for 60 minutes.

7) After the completion of the Neurobic Exercise Program, the MMSE - Thai 2002 and the Memory tests were performed.

\section{Data analysis}

The data were analyzed as follow:

1) Using descriptive statistics in a Demographic Data

2) Compare the differences of means scores before and after receiving Neurobic Exercise Program of the experimental group with Dependent T-Test for Paired Samples.

\section{Results}

The results revealed that: After receiving the Neurobic Exercise program, the average memory scores of the experimental group were statistically and significantly higher than before receiving as presented in Table 1, 2, 3 and 4.

Table 1. Comparing the Difference in Mean Memory Score for the Experimental Group Using the Mini-Mental State Examination - Thai 2002.

\begin{tabular}{llccc}
\hline \multirow{2}{*}{ Experimental group } & \multicolumn{3}{c}{ Memory score } & t \\
\cline { 2 - 4 } & $\bar{x}$ & S. D & \multirow{2}{*}{-6.876} & $.000^{* * *}$ \\
\hline Before receiving theNeurobic Exercise Program & 14.77 & 4.36 & 3.99 & \\
After receiving theNeurobic Exercise Program & 20.14 & & \\
\hline
\end{tabular}

***p $<.001$.

The data on table 1 reveals that the average memory scores of the experimental group after receiving the Neurobic Exercise were statistically and significantly higher than before receiving the Neurobic Exercise Program at a level of $p<.001$.

Table 1. Comparing the Difference in Mean Memory Score for the Experimental Group Using the Memory Test

\begin{tabular}{|c|c|c|c|c|}
\hline \multirow[b]{2}{*}{ Experimental group } & \multicolumn{2}{|c|}{ Memory score } & \multirow[b]{2}{*}{$\mathbf{t}$} & \multirow{2}{*}{$\boldsymbol{P}$} \\
\hline & $\bar{x}$ & S. D & & \\
\hline Before receiving theNeurobic Exercise Program & 27.68 & 8.68 & \multirow{2}{*}{-7.750} & \multirow{2}{*}{$.000 * * *$} \\
\hline After receiving theNeurobic Exercise Program & 35.95 & 5.62 & & \\
\hline
\end{tabular}

$* * * p<.001$.

The data on Table 2 reveals that the average memory scores of the experimental group after receiving the Neurobic Exercise Program were statistically and significantly higher than before receiving the Neurobic Exercise Program at a level of $p<.001$. 
Table 3. Comparing the Differences in Mean Memory Score on Memory Aspects for the Experimental Group Using the Mini-Mental State Examination - Thai 2002.

\begin{tabular}{lll}
\hline \multirow{2}{*}{ Aspects of Memory } & \multicolumn{2}{c}{ Scores } \\
\cline { 2 - 3 } & Before experiment & After experiment \\
\hline Orientation for time (5marks) & 2 & 4.14 \\
Orientation for place (5 marks) & 2.14 & 3.72 \\
Registration (3 marks) & 2.23 & 2.55 \\
Attention / calculation (5 marks) & 1.09 & 1.55 \\
Recall (3 marks) & 1.18 & 1.41 \\
Naming (2 marks) & 1.95 & 2.00 \\
Repetition (1 mark) & 0.23 & 0.77 \\
Verbal command (3marks) & 2.95 & 3.00 \\
Written command (1 mark ) & 0.59 & 0.59 \\
Writing (1 mark) & 0.36 & 0.36 \\
Visuoconstruction (1 mark) & 0.05 & 0.05 \\
Average scores & 14.77 & 20.14 \\
\hline
\end{tabular}

The data on Table 3 reveals that the average memory scores of the experimental group in aspects of orientation for time, orientation for place, registration, attention / calculation, recall and repetition after receiving the Neurobic Exercise were higher than before receiving the Neurobic Exercise Program.

Table 4. Comparing the Differences in Mean Memory Score on Memory Aspects for the Experimental Group Using the Memory Test.

\begin{tabular}{llc}
\hline \multirow{2}{*}{ Aspects of Memory } & \multicolumn{2}{c}{ Scores } \\
\cline { 2 - 3 } & Before experiment & After experiment \\
\hline Face and name recall (5 marks ) & 1.41 & 3.95 \\
Digits recall (5 marks) & 3.77 & 4.86 \\
The words clustering (5 marks) & 4.09 & 4.68 \\
Drawing the assigned (5 marks) & 2.32 & 2.32 \\
Scanning main ideas from reading(10 marks) & 3.91 & 5.87 \\
Scanning main ideas from listening(10 marks) & 3.82 & 4.50 \\
Items storing (10 marks) & 8.36 & 9.77 \\
Average scores & 27.68 & 35.95 \\
\hline
\end{tabular}

The data on Table 4 reveals that the average memory scores of the experimental group in aspects of face and name recall, digits recall, the words clustering, scanning main ideas from reading, scanning main ideas from listening and items storing after receiving the Neurobic Exercise were higher than before receiving the Neurobic Exercise Program.

\section{Discussion}

Hypothesis: Memory scores of the experimental group after receiving the Neurobic Exercise to be higher than before receiving the Neurobic Exercise. This hypothesis has been supported as the average memory scores of the experimental group after receiving the Neurobic Exercise Program were statistically and significantly higher than before receiving the Neurobic Exercise Program at a level of $p<.001$. In addition, the researcher compared the differences in mean memory score on memory aspects for the experimental group using the Mini-Mental State Examination method - Thai 2002 and the Memory test. As the results of most of the memory scores on memory aspects of Mini-Mental State Examination were found to be higher except on written command, writing and visuoconstruction. The memory scores in memory aspects of 
the Memory test too were found to be higher except on drawing the assigned. In the Neurobic Exercise Program, the experimental group was encouraged to use six senses of sight, hearing, smelling tasting, touching and emotional sense and breaking a routine activity in an unexpected way to strengthen of nerve cell stimulation ${ }^{[12]}$. Neurobic Exercise Program in each sense can activate brain functions and nerve connections as mentioned below:

1) Using the sense of vision by closing the eyes, identifying items and body language. These will stimulate occipital lobe, which processes visual information and diencephalon (thalamus and hypothalamus) that the activities in the cortex which is important in relaying sensory information to the cerebral cortex. All sensory information (except olfactory information) will filter out unnecessary sensory information and synchronize important sensory information in the thalamus ${ }^{[13]}$.

2) Using the sense of hearing by listening to music, facilitates temporal lobe and limbic system which are involved in emotion, motivation, and emotional association with memory. The limbic system plays its role in the formation of memory by integrating emotional states with stored memories of physical sensations ${ }^{[13]}$.

3) Using sense of smelling by smelling essential oils, aroma and herb such as lemon grass, garlic, red onions, lime, mangos, roses, jasmines, coffee and tea, which are familiar to the elders. It helps activate temporal lobe that is involved with the senses of smell and sound. The processing of semantics in both speech and vision and plays a key role in the formation of long-term memory. Deep inside the temporal lobe is the region of the limbic system, which includes hippocampus, amygdala, thalamus and hypothalamus which are of particular relevance to the processing of memory ${ }^{[13]}$. The hippocampus acts as a temporary transit point for long-term memories as it does not store information ${ }^{[13]}$. However, it is essential to the consolidation of information from short-term to long-term memory. It is also an area that produces the chemical acetylcholine which facilitates learning and memory ${ }^{[3,12]}$. Moreover, amygdala also performs a primary role in the processing and memory of emotional reactions. Thus, the smells may be more quickly and more strongly associated with memories than the other senses, and memories of a smell may persist for longer ${ }^{[13]}$.

4) Using sense of taste by tasting dessert (Tong yod and Kanom-chun), lime, sugar, salt, coffee, tea and cookie. It helps to stimulate parietal lobe, which involved in integrating sensory information from various senses, cognition and information processing of time, place orientation and memory.

5) Using the sense of touch by massaging, clay molding, objects identifying by touching such as coins, spoons, glasses, pens, rulers, combs, tooth brushes, etc. Those are the objects which the older adults have been using regularly. It activates frontal and parietal lobe. Frontal lobe is involved in conscious thought and higher mental functions such as decision-making, particularly in that part of the frontal lobe known as the prefrontal cortex. It also plays an important part in processing short-term memories and retaining longer term memories. These are memories associated with emotions derived from input from the limbic system. In addition, the parietal lobe also plays a role in sensory regulation ${ }^{[13]}$.

6) Using of the emotional sense combined in five senses such as play card, listen to music, massage and smell aroma etc. Emotional sense will motivate diencephalon, especially hypothalamus, which regulate emotion and encode memory by laying down a memory attention. It is also stimulate limbic system, which plays a role in the formation of memory by integrating emotional states with stored memories of physical sensations, it included amygdala and hippocampus. The amygdala is responsible for determining what memories are stored and where the memories are stored in the brain and plays a role in the modulation of emotional memory consolidation. The hippocampus involved in memory forming, organizing, and storing ${ }^{[15]}$. It is important in forming new memories and connecting emotions and senses, such as smell and sound. The hippocampus acts as a memory indexer by sending memories out to the appropriate part of the cerebral cortex for long-term storage and retrieving them when necessary ${ }^{[15]}$. 
During the session of Neurobic Exercise Program, the participants received the program to use senses in combining two or more senses a day. Thus, the combination of two or more senses stimulated the function of brain, which involved with memory process, including the frontal, parietal, temporal, occipital lobe, diencephalon and limbic system. Thus, the Neurobic exercise program by using various combinations of physical senses stimulated the function of neural network ${ }^{[12]}$ which involved with short-term and long term memory ${ }^{[14]}$ of the participants. Most types of memories are stored in the cortex because almost $90 \%$ of the neurons are located in the cerebral cortex which is connected to cerebellum and spinal cord $^{[13]}$. In the medial temporal lobe of cerebral cortex where the hippocampus is located and the amygdala is located adjacent to it. These two parts play a key role in short-term memory. Memory begins with the sense taking in a stimulus at the cerebral cortex and transformed into sensory memory. If the information is repeated, then it will be transferred to limbic system in hippocampus and stored as a working or short term memory ${ }^{[13,14]}$. Short-term memory will hold a piece of information temporarily, which would lead to long-term memory later. The permanent storage in long -term memory can be facilitated by the repetition of the information ${ }^{[13,14]}$.

Moreover, Neurobic Exercise Program presented the brain with non- routine or unexpected experiences such as brushing teeth, writing, combing hair and eating with unfamiliar hand or changing the sequence of daily activities. For example: having dinner before taking a shower or changing the places where they usually had their meals. This program can be done anywhere, anytime in offbeat, fun and easy ways. This helps in balancing the brain functions and activating the underused nerve pathways and connections ${ }^{[12]}$. As new experiences accumulate, the brain creates more and more connections and pathways, and it could help re-wire itself by re-routing connections and re-arranging its organization ${ }^{[12,13]}$. It can also prevent the loss of the brain from under using ${ }^{[12]}$. All members of the experimental group were females as Mitraparpsongkoh Foundation located in Pathum Thani Province in Thailand, accommodates only elderly females. Even though the incidence rates of dementia were found to be higher in elderly women than the elderly men who is significantly related to the effects of hormones and life expectation of women ${ }^{[16,17]} .86 .4 \%$ of the experimental group the average age was 77.2 years. This indicated that they are likely to lose neurons less than at old age. As the brain shrinks in size of people who are older than 85 years and $35 \%$ of them are suffering from dementia ${ }^{[1]}$. In addition, this program was divided into 14 sessions on every 2 days continuously for 4 weeks. Thus, the memory can be restored in short memory and eventually transform to long-term memory by repeating the 14 sessions of Neurobic exercise program.

Although there are no sufficient studies recorded in the past to support the specific practices of Neurobic exercise, it does not mean that Neurobic exercise is of no value ${ }^{[18]}$. However, Neurobic exercise is a mental exercise that can reduce the risk of dementia ${ }^{[19]}$. Mental exercise includes crossword puzzles, playing chess, board games, musical instruments, games, reading and dancing. These activities can reduce the rates of memory decline, same as the study of Leisure activities and the risk of dementia in the elderly ${ }^{[19]}$. It is also found a reduction of 7 percent in the risk of dementia on elderly participants who were involved in leisure activities such as reading, doing crossword puzzles, playing board games, and playing cards for once a week ${ }^{[19]}$. In addition, the study of cognitive activity and the cognitive morbidity of Alzheimer disease in the older people found the elderly people who participated in cognitive activities such as reading book, magazines, newspapers, playing games, cards and crosswords, watching TV and going to a museum had delayed dementia onset after the six years of evaluation by using cognitive activity scale ${ }^{[20]}$. Therefore, participating in cognitive activity frequently can stimulate different parts of the brain involved in memory and information processing. These parts of the brain become more efficient in maintaining memory ${ }^{[20]}$, even though the strength evidence to support the cognitive exercises remains low ${ }^{[21]}$. However, the systematic review of cognitive exercises found that, it may be effective at enhancing cognitive outcomes to a person who has mild cognitive impairment ${ }^{[21]}$.

This Neurobic exercise program was not only using all senses, but also encouraged the experimental group participated in playing cards, digits recalling, mind calculating, asking time and place, phrase repetitionand picture naming. All these activities are the same as mental exercise or cognitive activities. Therefore, activities also can facilitate frontal and parietal lobe, diencephalon and limbic system which involved with memory process. In addition, Neurobic Exercise Program was 
also a fun activity. Then the participants felt relaxed and happy whilst leading to improve their memory retention as the brain circuits were alerted and activated.

Therefore, it can be concluded that Neurobic Exercise Program improved the memory retention in the elderly with dementia with statistical significance.

\section{Limitations of this study}

1) This research supposed to be an introductory research in the elderly with dementia. Therefore, the design of this research is Quasi-experimental research One Group Pretest-Posttest without control group and the participants were also selected by a purpose sampling. Thus, the lack of random assignments in this designed method can be defined as a limited design.

2) The participants of this research could be only suffering from a state of mild dementia which diagnosed by using Mini-Mental State Examination - Thai 2002. But they were not subjected to the method of Diagnostic and Statistical Manual of Mental Disorders.

3) This study focused only on older females who are suffering from dementia. Thus, the sample might not represent all older people who are suffering from dementia.

4) The activities in this Neurobic exercise program were selected only on safe activities for the participants.

5) This research did not test the sustainability of the memory after participating in Neurobic exercise program continuously.

\section{Applications}

1) Nursing care- nurses are required to be trained in Neurobic Exercise Program by applying six physical senses as well as non- routine experiences regularly whilst they provided nursing care to the elderly people who are suffering from dementia.

2) Nursing administration- the results of this study can be established as a nursing standard in improving memory deterioration and also in a multidisciplinary team to create a care map and a clinical pathway that could ensure the quality control in nursing care.

3) Nursing academy - these findings may be integrated to the elderly care subject in teaching nursing students in enhancing memory impairment the dementia elders.

4) Future research- this could be used as base information on further research in the elderly with dementia or others suffering from similar disorders in improving the quality of their life.

\section{References}

[1] Jriangprasert C. Alzheimer and Dementia (2 ${ }^{\text {nd }}$ ed). Bangkok: NatangsulokkwangPublisher; 2006.

[2] Sriwatanakul K. How to keep away from dementia ( $1^{\text {st }}$ ed). Bangkok: Siam sport syndicate; 2002.

[3] Myers EC. Memory loss and the brain. The new letter of the Memory Disorders Project. 2006. Available from URL: http://www.memorylossonline.com/glossary/memory.html.

[4] Kairith O, Chansirikan R. Thai people are vulnerable for Dementia. The Newsletter Post 2009 March (vol. 3). 
[5] Pinquart M, Sorensen S. Associations of stressors and uplifts of caregiving with caregiver burden and depressive mood: A meta-analysis. Journal of Gerontology. 2003; 58 (2): 112-128.

[6] Health Information System Development Office 2009. Thaihealth Report 2007. Available from: http://www.hiso.or.th.

[7] World Health Organization. 10 Fact of dementia. WHO, 2012. Available from: www.who.int/features/factfiles/dementia/en/index.html.

[8] Kenny T, Kenny B, Allen H, Bonsall MA, Cox PJ, Draper R, et al.Memory Loss and Dementia 2012. Available from: http://www.patient.co.uk/health/Memory-Loss-and-Dementia.htmhttp://dx.doi.org/10.1093/geronb/58.2.P112

[9] Tadaka E, Kanagava K. A randomized controlled trial of a group care program for community-dwelling elderly people with dementia. Japan Journal of Nursing Science. 2004; 1: 19-25.

[10] Chaiwong W. Effectiveness of the Memory Training Program for the Elderly [dissertation]. Chiang Mai: ChiangMai Univ., 2005.

[11] Nicola TL, Cox LK, Flicker L,Foster KJ, Bockxmeer MF, Xiao J, et al. Effect of physical activity on cognitive function in older adults at risk for alzheimer disease: A Randomized Trial FREE . JAMA. 2008; 300(9):1027-1037.

[12] Katz CL, Rubin M. Keep your brain alive: 83 Neurobic Exercises to help prevent memory loss and increase mental fitness. New York: Workman Publishing Company; 1999.

[13] Mastin, L. The Human Memory 2010. Available from: http://www.human-memory.net.

[14] Atkinson RC, Shiffrin RM. Memory and cognition. In S. Warchel\& W. Shebilsk (Eds.), Psychology: Principles and application ( $2^{\text {nd }}$ ed.). (pp.219-258). New Jersy: Prentice Hall; 1977. http://dx.doi.org/10.1111/j.1742-7924.2004.00006.x

[15] Bailey R. Hippocampus 2012. Available from URL: http://biology.about.com/od/anatomy/p/hippocampus.htm

[16] Institute of Research Foundation and Development for older Thai people. Health report in 2008 of older Thai people. Available from: www.ryt9.com/s/cabt/800885.

[17] Bamford, S-M. Women and Dementia- Not forgotten 2011. London: The International Longevity Centre-UK.

[18] Dustin B. Improve Brain Health with Neurobics. Emergency Medicine News. 2010; 32(12).

[19] Verghese J, Lipton BR, Katz JM, Hall BC, Derby AC, Kuslansky G, et al. Leisure activities and the risk of dementia in the elderly.The New England Journal Medicine. 2003; 348: 2508-2516. PMid:12815136 http://dx.doi.org/10.1056/NEJMoa022252

[20] Wilson RS, Barnes LL, Aggarwal NT, Boyle PA, Hebert LE, Mendes de Leon CF \& Evans DA. Cognitive activity and the cognitive morbidity of Alzheimer disease. Neurology. 2010; 75: 990-996.

[21] Gates NJ, Sachdev PS, Fiatarone Singh MA, Valenzuela M. Cognitive and memory training in adults at risk of dementia: A Systematic Review. BMC Geriatrics; 2011. Available from: http://www.biomedcentral.com/1471-2318/11/55. 